\title{
Avaliação das propriedades mecânicas e morfológicas de compósitos de PEAD com pó de Pinus taeda e alumina calcinada
}

\section{Evaluation of the mechanical and morphological properties of HDPE composites with powdered Pinus taeda and calcined alumina}

\author{
Karine Grison ${ }^{1}$, Taís Caroline Turella ${ }^{1}$, Lisete Cristine Scienza² e Ademir José Zattera ${ }^{1 *}$ \\ 'Programa de Pós-Graduação em Engenharia de Processos e Tecnologias, Universidade de \\ Caxias do Sul - UCS, Caxias do Sul, RS, Brasil \\ ${ }^{2}$ Departamento de Materiais, Universidade Federal do Rio Grande do Sul - UFRGS, \\ Porto Alegre, RS, Brasil \\ *ajzattera@terra.com.br
}

\section{Resumo}

Neste estudo foram desenvolvidos compósitos utilizando PEAD, pó de madeira (Pinus taeda), alumina calcinada e dois diferentes tipos de agentes compatibilizantes para avaliação das propriedades morfológicas e mecânicas dos mesmos. Para aumentar a interação entre a matriz polimérica e o pó de madeira foram utilizados $2 \%$ de polietileno graftizado com anidrido maleico em todas as formulações. Para efeito comparativo foi desenvolvida uma formulação com viniltrietoxisilano como compatibilizante para a alumina calcinada. O teor de cargas variou de $4 \%$ a $33 \%$ para os compósitos de carga única e mantiveram o percentual de $28 \%$ para os compósitos com as duas cargas. A interação entre a matriz polimérica e as cargas, proporcionada pelo agente compatibilizante anidrido maleico, foi observada nas micrografias da interface da matriz/carga. A utilização do silano não proporcionou efeito adicional nas propriedades mecânicas dos compósitos. Os compósitos isentos de alumina apresentaram maior resistência à tração, porém na resistência à flexão a presença da alumina contribuiu para o aumento desta propriedade provavelmente devido à pequena interação existente entre a interface do seu grão e a matriz polimérica.

Palavras-chave: compósito, PEAD, pó de madeira, alumina calcinada, anidrido maleico.

\begin{abstract}
This work aims at the evaluation of morphological and mechanical properties of HDPE composites developed with wood flour (Pinus taeda), calcined alumina and two different types of compatibilizing agents. In order to improve the interaction between the polymer matrix and wood flour $2 \%$ maleic anhydride-grafted polyethylene was used in all formulations. For comparison a formulation with triethoxyvinylsilane as compatibilizer for the calcined alumina was developed. The filler content ranged from $4 \%$ to $33 \%$ for the single filler composite while the percentage of $28 \%$ was kept as such for the two-fillers composites. The interaction between the polymer matrix and fillers, provided by the maleic anhydride compatibilizer agent, could be observed in the micrographs of the matrix / filler interface. The silane did not improve the mechanical properties of the composite. Free alumina composites showed higher tensile strength, but concerning flexural strength the presence of alumina contributed to increased values in this property. This was probably caused by the reduced interaction between the interface of its grain and the polymer matrix.
\end{abstract}

Keywords: composite, HDPE, wood flour, calcined alumina, maleic anhydride.

\section{Introdução}

Os materiais compósitos são uma alternativa em substituição aos materiais utilizados em setores que abrangem desde a construção civil à indústria automobilística, aliando propriedades como baixa densidade, boas propriedades mecânicas, facilidade de moldagem e elevada resistência à corrosão e à fadiga ${ }^{[1,2]}$. Sua utilização pode ser voltada à redução do custo do produto final e/ou à melhora em alguma propriedade específica como: mecânica, térmica, acústica, elétrica ou ótica, fazendo com que os materiais compósitos ganhem especial destaque na indústria ${ }^{[3,4]}$. 
Em materiais compósitos são empregados polímeros, cerâmicas e metais como matriz e utilizam-se fibras/pós vegetais ou minerais, como fase dispersa ${ }^{[5-8]}$. Quando os polímeros são utilizados como matriz os mais usuais são o polietileno de baixa e alta densidade (PEBD e PEAD), o polipropileno (PP), o policloreto de vinila (PVC), o poliestireno (PS), o acrilonitrila-butadieno-estireno (ABS) e o nylon (PA) $)^{[9,10]}$.

Entre as cargas utilizadas para conferir um melhor desempenho às propriedades dos compósitos estão as de natureza mineral (carbonatos, silicatos, argilas e outras) ${ }^{[11]}$, ou vegetal (sisal, juta, algodão, madeira e outras). A carga natural vegetal possui a vantagem de ser uma fonte renovável, contudo poderá apresentar variações em suas propriedades químicas devido às condições climáticas e regionais onde se dá o cultivo e também ao processamento das mesmas ${ }^{[12]}$. Sua polaridade dificulta a interação destes com a matriz polimérica apolar, sendo necessário o uso de agentes compatibilizantes aumentando a área interfacial entre matriz/carga objetivando melhorias significativas nas propriedades do compósito ${ }^{[13]}$. As propriedades de resistência à tração, flexão e módulo de elasticidade podem atingir valores superiores com o uso de agente compatibilizante ${ }^{[14,15]}$ e com a seleção correta do tipo e do percentual de carga vegeta[ ${ }^{[16]}$. Alguns dos compatibilizantes mais utilizados em compósito termoplástico com carga vegetal são os baseados em anidrido maleico, silanos ou peróxidos de polietileno, promovendo uma boa adesividade interfacial entre as fibras e a matriz ${ }^{[13,17]}$.

Para este trabalho utilizaram-se PEAD, pó de Pinus taeda, alumina calcinada, anidrido maleico graftizado e viniltrietoxisilano com o objetivo de avaliar a influência dos diferentes percentuais de carga vegetal e mineral nos compósitos e o efeito dos agentes de acoplamento nas propriedades mecânicas dos compósitos.

\section{Materiais e Métodos}

\subsection{Materiais}

O polietileno de alta densidade (PEAD) grade ES 6004, fornecido pela Braskem, possui índice de fluidez de $0,35 \mathrm{~g} / 10 \mathrm{~min}\left(190{ }^{\circ} \mathrm{C} / 2,16 \mathrm{~kg}\right)$ e densidade $0,96 \mathrm{~g} / \mathrm{cm}^{3}$. $\mathrm{O}$ agente compatibilizante utilizado para a carga vegetal foi o polietileno de alta densidade graftizado com anidrido maleico (PE-g-MA), especificação 3029 Polybond, da Chemtura; e para a carga mineral o agente compatibilizante viniltrietoxisilano (VTES), 97\% de pureza, da empresa Sigma-Aldrich. As tábuas de Pinus taeda foram fornecidas por uma empresa do ramo de materiais de construção de Caxias do Sul e a alumina calcinada A-2 foi fornecida pela empresa Alcoa S/A, possuindo $99,2 \%$ de $\mathrm{Al}_{2} \mathrm{O}_{3}$ e alto grau de hidrofobicidade.

\subsection{Preparação das amostras}

As tábuas de Pinus taeda foram serradas, moídas em moinho de facas e classificadas utilizando as peneiras (Escala Tyler) com malhas 35, 48, 65, 100 e 150. Posteriormente foram misturadas às formulações em percentuais de 14,19 , 24 e 33\%, sendo cada percentual elaborado com a proporção granulométrica apresentada na Tabela 1 . A alumina foi tratada com $0,5 \%$ de VTES em solução $95 \%$ de etanol e agitada por 2 horas. A amostra permaneceu em repouso por 16 horas. Após a remoção do sobrenadante a alumina tratada foi seca em estufa por 5 horas a $90^{\circ} \mathrm{C}$ antes de ser adicionada às formulações nos percentuais de 4, 9,14 e $33 \%(\mathrm{~m} / \mathrm{m})$ e o percentual dos grãos obtidos na classificação granulométrica encontra-se na Tabela 2.

As Tabelas 3 e 4 apresentam as formulações de compósitos desenvolvidas. As amostras foram secas em estufa por 24 horas, a $80{ }^{\circ} \mathrm{C}$ e processadas em extrusora duplarrosca marca MH Equipamentos L/D 32, com rosca

Tabela 1. Percentual das diferentes granulometrias de pó de Pinus taeda utilizadas nas formulações dos compósitos confeccionados com pó de madeira.

\begin{tabular}{ccccc}
\hline Malha & $\mathbf{4 8}$ & $\mathbf{6 5}$ & $\mathbf{1 0 0}$ & $\mathbf{1 5 0}$ \\
\hline \% usado & 36 & 36 & 14 & 14 \\
\hline
\end{tabular}

Tabela 2. Percentual das diferentes granulometrias de alumina utilizadas nas formulações dos compósitos.

\begin{tabular}{|c|c|c|c|c|c|c|}
\hline Tyler & $+65 /-100$ & $+100 /-150$ & $+150 /-200$ & $+200 /-270$ & +270 /-fundo & +Fundo \\
\hline massa (g) & 0 & 0,33 & 1,07 & 0,53 & 0,06 & 0,05 \\
\hline$\%$ em massa & 0 & 16,33 & 52,39 & 26,17 & 2,85 & 2,26 \\
\hline
\end{tabular}

Tabela 3. Percentuais dos componentes utilizados nas formulações dos compósitos de carga única.

\begin{tabular}{lccccccc}
\hline \multicolumn{1}{c}{ Materiais* } & 9A & 9A/2MA & 9A/0,5S & 14M/2MA & 14A/2MA & 33M/2MA & 33A/2MA \\
\hline PEAD & 91 & 89 & 90 & 84 & 84 & 65 & 65 \\
PE-g-MA (MA) & 0 & 2 & 0 & 2 & 2 & 2 & 2 \\
VTES (S) & 0 & 0 & 0,5 & 0 & 0 & 0 & 0 \\
Pó de madeira (M) & 0 & 0 & 0 & 14 & 0 & 33 & 0 \\
Alumina (A) & 9 & 9 & 9 & 0 & 14 & 0 & 33 \\
\hline
\end{tabular}

*Valores expressos em \% em massa. A simbologia adotada refere-se a quantidade e natureza do material adicionado. Ex: 9A/2MA significa 9\% de alumina e $2 \%$ de anidrido maleico. 
Tabela 4. Percentuais dos componentes utilizados nas formulações dos compósitos com duas cargas.

\begin{tabular}{lccc}
\hline \multirow{2}{*}{ Materiais* $^{*}$} & \multicolumn{3}{c}{ Compósitos } \\
\cline { 2 - 4 } & $\mathbf{2 4 M / 4 A / 2 M A}$ & $\mathbf{1 9 M / 9 A} / \mathbf{2 M A}$ & $\mathbf{1 4 M / 1 4 A / 2 M A ~}$ \\
\hline PEAD & 70 & 70 & 2 \\
PE-g-MA (MA) & 2 & 2 & 14 \\
Pó de madeira (M) & 24 & 19 & 14 \\
Alumina (A) & 4 & 9 & \\
\hline
\end{tabular}

*Valores expressos em \% em massa.

de $20 \mathrm{~mm}$, utilizando o perfil de temperatura de $145^{\circ} \mathrm{C}$, $170{ }^{\circ} \mathrm{C}, 180^{\circ} \mathrm{C}, 180^{\circ} \mathrm{C}, 178{ }^{\circ} \mathrm{C}, 165^{\circ} \mathrm{C}, 180^{\circ} \mathrm{C}, 185^{\circ} \mathrm{C}$ e $185^{\circ} \mathrm{C}$ da alimentação até a matriz, e com velocidade de rosca de $200 \mathrm{rpm}$. As amostras obtidas da extrusão foram secas em estufa por 24 horas, a $80{ }^{\circ} \mathrm{C}$ e posteriormente foram injetados os corpos de prova para ensaios numa injetora Himaco, modelo LHS 150-80, utilizando-se perfil de temperatura de $190{ }^{\circ} \mathrm{C}, 180{ }^{\circ} \mathrm{C}$ e $170{ }^{\circ} \mathrm{C}$ e velocidade de rosca de $60 \mathrm{rpm}$ na injetora.

\subsection{Caracterização}

A avaliação morfológica dos compósitos e das matériasprimas foi realizada com um microscópio eletrônico de varredura da marca Shimadzu, modelo SSX-550 Superscan. Os corpos de prova utilizados foram fraturados criogenicamente e recobertos com uma fina camada de ouro antes da análise. Para os ensaios mecânicos foi utilizada uma máquina universal de ensaio EMIC DL 2000, segundo a norma ASTM D 638:10 para o ensaio de resistência à tração, com velocidade de $10 \mathrm{~mm} \cdot \mathrm{min}^{-1}$. Os ensaios de flexão foram realizados conforme a norma ASTM D 790:10, utilizando-se célula de carga de $100 \mathrm{kgf}$ e velocidade de $1,5 \mathrm{~mm} \cdot \mathrm{min}^{-1}$. O ensaio de impacto IZOD, com entalhe, pêndulo de $1 \mathrm{~J}$ e velocidade de $3,5 \mathrm{~m} \cdot \mathrm{s}^{-1}$ foi realizado em equipamento da CEAST, modelo Resil 25, conforme a norma ASTM D 256:10.

\section{Resultados e Discussões}

\subsection{Microscopia Eletrônica de Varredura (MEV)}

Na Figura 1a visualiza-se uma boa adesão na interface madeira/PEAD para o compósito com $33 \%$ de pó de madeira, que influencia de forma positiva nas propriedades mecânicas. Pela análise da Figura $1 \mathrm{~b}$ pode-se visualizar um grão de alumina e seu interior, formado por um aglomerado de inúmeras partículas de alumina. Observa-se que na Figura 1c o interior das partículas não apresenta a presença da matriz polimérica tornando o grão um ponto frágil na estrutura do compósito.

A Figura 2 mostra as micrografias da região fraturada para os compósitos contendo duas cargas (pó de madeira e alumina) em matriz de PEAD modificada com anidrido maleico.

Na Figura 2a e b observa-se a boa adesão interfacial entre a matriz de PEAD e a fase dispersa de madeira. A análise da figura mostra que não houve descolamento na área interfacial matriz/madeira e ocorreu o rompimento da partícula da madeira próximo à superfície da matriz, corroborando a afirmação da boa adesão da carga à matriz.
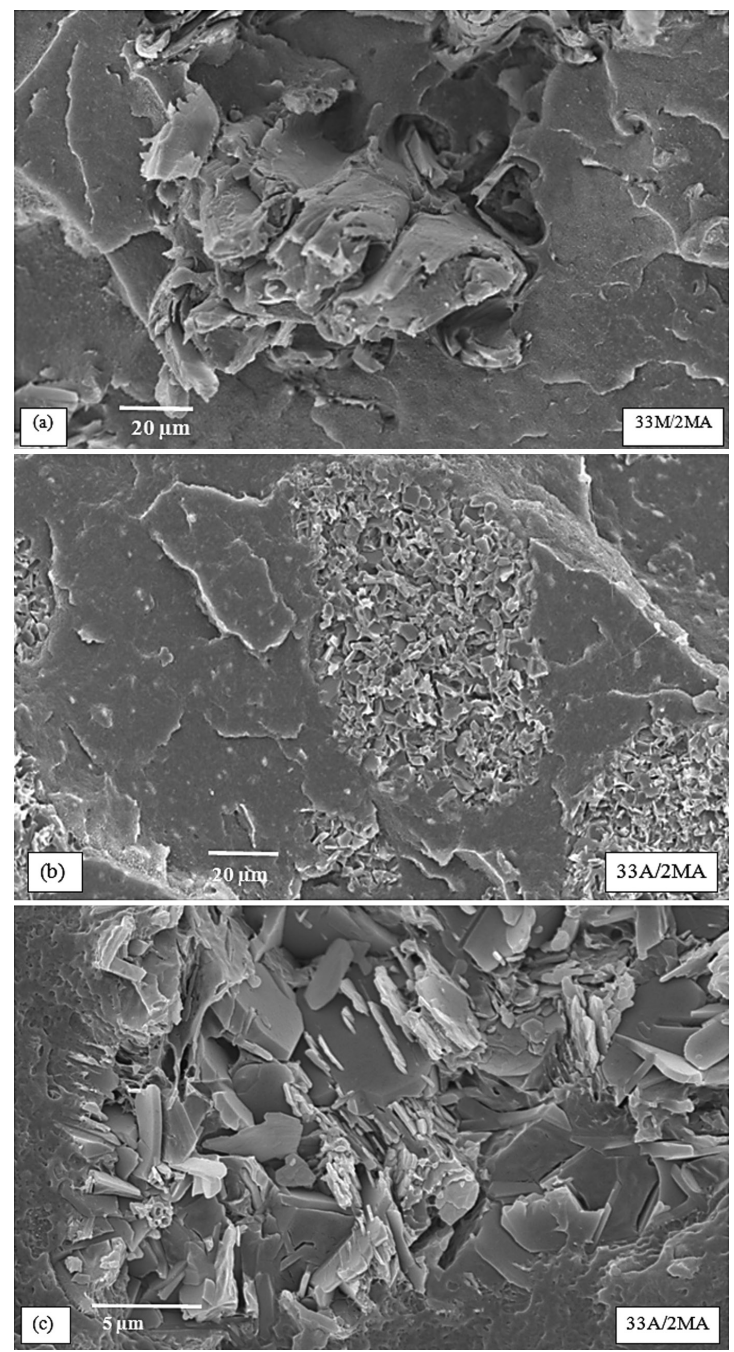

Figura 1. Micrografias da região fraturada (a) e (b) com aumento de 1000x para os compósitos de fase única utilizando percentual de $33 \%$, e PEAD-g-MA como compatibilizante e micrografia (c) com aumento de 6000x para o interior do grão de alumina.

$\mathrm{Na}$ Figura 2c visualiza-se ambas as cargas na mesma região de análise do microscópio. Observa-se que a matriz polimérica envolve a madeira $(m)$ e interpenetra em suas camadas, isto não se percebe com a alumina $(a)$, onde a matriz encontra-se somente no entorno de seu grão. Esta baixa interação observada pode influenciar negativamente nas propriedades mecânicas do compósito pelo fato de não haver adesividade interna entre as partículas de alumina que constituem o grão. 

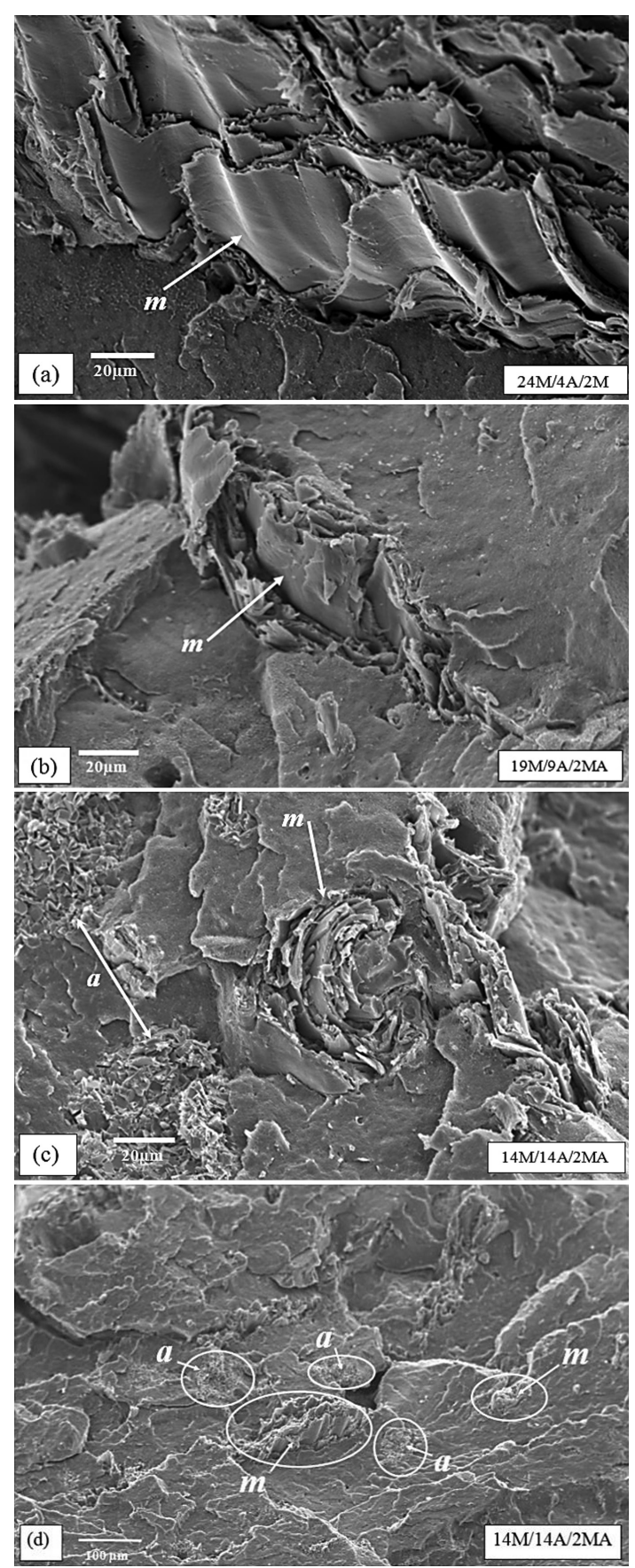

Figura 2. Micrografias para os compósitos utilizando duas fases dispersas e matriz de PEAD modificado com o agente compatibilizante PEAD-g-MA. Onde $a$ é alumina e mé madeira.

\subsection{Resistência à tração}

Analisando a Tabela 5 observou-se que nas amostras 9A/2MA e 9A/0,5S não houve alteração significativa nos valores para a resistência à tração com o uso de diferentes agentes compatibilizantes. Por outro lado houve um acréscimo das propriedades mecânicas com o uso dos agentes compatibilizantes em relação ao PEAD.
As amostras contendo pó de madeira apresentaram valores de resistência à tração superior ao valor do PEAD virgem, o que pode indicar uma boa interação da madeira com o polímero proporcionado pela adição do agente compatibilizante $\mathrm{MA}^{[17]}$. O maior teor de madeira (33\%) aliado à boa interação madeira/HDPE, conforme visto nas micrografias anteriores (Figuras 1a e 2a), auxilia na dissipação da energia da matriz para a carga, aumentando o valor da propriedade de resistência à tração em $52 \%$ neste compósito. A amostra que apresentou o melhor desempenho foi o compósito com 33\% de madeira (formulação 33M/2MA), houve um aumento da propriedade de resistência à tração e do módulo elástico. Por outro lado, a adição da alumina ocasiona uma diminuição das propriedades mecânicas devido a pouca interação entre as pequenas partículas que formam o grão de alumina observado na Figura 1c. Verifica-se um aumento gradual na resistência à tração à medida que se aumenta o percentual de pó de madeira utilizado nas amostras, o que corrobora o fato da madeira agir como um reforço distribuindo as tensões exercidas sobre a matriz ${ }^{[3]}$ e à boa interação com a matriz polimérica observadas nas Figuras 1 e 2 .

\subsection{Resistência à flexão}

Analisando os resultados da Tabela 6 pode-se realizar um comparativo do desempenho entre as formulações de compósitos utilizando anidrido maleico (9A/2MA) e de compósitos utilizando silano $(9 \mathrm{~A} / 0,5 \mathrm{~S})$ no ensaio de resistência à flexão.

Nesta comparação observou-se um desempenho superior para os compósitos com anidrido maleico. A partir da análise do desempenho dos agentes compatibilizantes contidos nas Tabelas 4 e 5, observou-se que, para as formulações de compósitos utilizados neste trabalho, o agente compatibilizante mais indicado foi o anidrido maleico. Este melhor desempenho do anidrido maleico pode ser explicado pelas reações desencadeadas pelo agente de acoplamento na interface madeira/matriz quando grupos hidroxila $(\mathrm{OH})$ da celulose reagem com o anidrido proporcionando maior ancoragem ao polímero e menor ação hidrofílica por parte da madeira ${ }^{[1,17]}$

A formulação de compósito que obteve o melhor desempenho quanto às propriedades de resistência à flexão foi a amostra contendo $24 \%$ de madeira e $4 \%$ de alumina (24M/4A/2MA), onde a menor quantidade de alumina consegue distribuir-se de maneira mais homogênea na matriz, posicionando-se nos espaços existentes entre uma partícula de madeira e outra conforme visualiza-se na Figura 2d.

Os compósitos contendo somente alumina obtiveram os menores valores para a resistência à flexão, confirmando que foi visualizado na micrografia do aglomerado de alumina que apresenta seu interior fragilizado, sem interação com a matriz polimérica, Figura $2 \mathrm{c}$.

\subsection{Resistência ao impacto}

Através da análise dos resultados contidos na Tabela 7 , comparando-se as amostras utilizando alumina e agentes compatibilizantes distintos, observou-se que o uso do agente compatibilizante anidrido maleico (MA) obteve resultado superior ao agente compatibilizante à base de silano (S). Nos compósitos constatou-se que a adição de alumina e/ou 
Tabela 5. Propriedades mecânicas de resistência à tração para as diferentes formulações de compósitos e PEAD.

\begin{tabular}{lccc}
\hline \multicolumn{1}{c}{ Amostra } & Resistência à Tração (MPa) & Deformação (\%) & Módulo Elástico (MPa) \\
\hline PEAD & $21,43 \pm 0,52$ & $19,61 \pm 1,11$ & $507,90 \pm 26,74$ \\
PEAD/2MA & $21,21 \pm 0,30$ & $15,73 \pm 0,40$ & $510,10 \pm 8,40$ \\
9A/2MA & $23,24 \pm 0,43$ & $18,84 \pm 0,79$ & $562,60 \pm 18,58$ \\
9A/0,5S & $22,98 \pm 0,76$ & $18,03 \pm 0,54$ & $600,90 \pm 28,78$ \\
14M/2MA & $27,16 \pm 0,35$ & $16,19 \pm 0,43$ & $699,90 \pm 22,95$ \\
14A/2MA & $22,89 \pm 0,39$ & $18,09 \pm 0,32$ & $594,60 \pm 17,70$ \\
33M/2MA & $32,39 \pm 0,69$ & $9,48 \pm 0,44$ & $1003,00 \pm 30,42$ \\
33A/2MA & $22,23 \pm 0,44$ & $13,63 \pm 0,45$ & $791,20 \pm 29,95$ \\
24M/4A/2MA & $30,22 \pm 0,51$ & $12,03 \pm 0,35$ & $829,00 \pm 27,64$ \\
19M/9A/2MA & $28,84 \pm 0,46$ & $12,35 \pm 0,26$ & $822,90 \pm 22,16$ \\
14M/14A/2MA & $27,10 \pm 0,33$ & $12,34 \pm 0,23$ & $835,20 \pm 20,78$ \\
\hline
\end{tabular}

Tabela 6. Propriedade mecânica de resistência à flexão para os compósitos e PEAD.

\begin{tabular}{lccc}
\hline \multicolumn{1}{c}{ Amostra } & Resistência à Flexão (MPa) & Deformação (\%) & Módulo Elástico (MPa) \\
\hline PEAD & $16,63 \pm 0,84$ & $6,85 \pm 0,38$ & $668,00 \pm 121,20$ \\
PEAD/2MA & $16,77 \pm 0,60$ & $6,51 \pm 0,40$ & $642,30 \pm 42,00$ \\
9A/2MA & $17,72 \pm 1,49$ & $6,80 \pm 0,19$ & $714,30 \pm 72,12$ \\
9A/0,5S & $14,63 \pm 1,79$ & $7,11 \pm 0,99$ & $519,20 \pm 157,10$ \\
14M/2MA & $28,25 \pm 0,93$ & $6,54 \pm 0,67$ & $1344,00 \pm 75,52$ \\
14A/2MA & $20,97 \pm 0,63$ & $6,74 \pm 0,16$ & $931,20 \pm 68,40$ \\
33M/2MA & $25,92 \pm 2,04$ & $6,42 \pm 0,24$ & $1181,00 \pm 153,90$ \\
33A/2MA & $16,25 \pm 0,39$ & $6,96 \pm 0,48$ & $644,30 \pm 29,47$ \\
24M/4A/2MA & $32,80 \pm 0,84$ & $6,09 \pm 0,37$ & $1761,00 \pm 89,40$ \\
19M/9A/2MA & $29,57 \pm 1,33$ & $6,30 \pm 0,20$ & $1674,00 \pm 155,60$ \\
14M/14A/2MA & $30,02 \pm 0,42$ & $6,55 \pm 0,28$ & $1642,00 \pm 53,70$ \\
\hline
\end{tabular}

Tabela 7. Propriedade mecânica de resistência ao impacto para os compósitos e o PEAD virgem.

\begin{tabular}{lc}
\hline \multicolumn{1}{c}{ Amostra } & Resistência ao Impacto (J/m) \\
\hline PEAD & NÃO ROMPEU * \\
PEAD/2MA & NÃO ROMPEU * \\
9A/2MA & $154,92 \pm 6,78$ \\
9A/0,5S & $92,22 \pm 18,71$ \\
14M/2MA & $111,62 \pm 4,67$ \\
14A/2MA & $119,52 \pm 2,80$ \\
33M/2MA & $71,12 \pm 5,42$ \\
33A/2MA & $77,26 \pm 3,34$ \\
24M/4A/2MA & $78,30 \pm 4,71$ \\
19M/9A/2MA & $77,61 \pm 4,67$ \\
14M/14A/2MA & $76,28 \pm 4,03$ \\
\hline
\end{tabular}

*Utilizando-se martelo de $1 \mathrm{~J}$.

pó de madeira ocasiona uma redução da resistência ao impacto, independente do teor utilizado. Estes resultados inferiores foram atribuídos à menor mobilidade molecular obtida à medida que aumenta-se o percentual das cargas nas formulações dos compósitos ${ }^{[18]}$.

Com relação à incorporação de pó de madeira em polímero termoplásticos, a redução da resistência ao impacto em relação ao polímero puro e o aumento da resistência à tração também foram constatados por outros pesquisadores ${ }^{[19]}$, sendo sugerido que este último efeito possa estar relacionado à característica da estrutura química da celulose, cuja macromolécula possui fortes interações de ligações de hidrogênio.
Os melhores resultados nos ensaios mecânicos observados com o uso do PEAD-g-MA, como agente de acoplamento para o pó de madeira na matriz polimérica, mostram que a presença de grupamentos de anidrido maleico no PEAD-g-MA auxiliam na interação das cadeias do polímero e celulose, contribuindo para uma melhor adesão entre a carga e a matriz ${ }^{[20]}$.

\section{Conclusão}

Através da análise morfológica foi constatada a existência de espaços vazios entre as partículas que constituem o grão de alumina, o que origina pontos frágeis quando incorporados à matriz polimérica. A superfície externa do grão apresentou boa adesão à matriz quando usado o compatibilizante anidrido maleico, o que contribuiu na propriedade de flexão para o compósito que utilizou o menor percentual de alumina 24M/4A/2MA.

Os resultados obtidos mostraram que os compósitos que contêm na sua formulação o agente compatibilizante à base de anidrido maleico possuem boas propriedades mecânicas, com valores superiores as do polímero puro bem como as dos demais compósitos contendo silano como agente compatibilizante.

$\mathrm{O}$ melhor resultado para a resistência à tração foi obtido com o compósito com a formulação 33M/2MA e para a resistência à flexão foi obtido com o compósito de formulação $24 \mathrm{M} / 4 \mathrm{~A} / 2 \mathrm{MA}$, demonstrando que a alumina influi negativamente nos esforços de tração, mas proporciona maior rigidez ao material, aumentando sua resistência à flexão. Para a resistência ao impacto observou-se que o aumento de carga 
de madeira e/ou alumina ocasiona uma menor resistência ao impacto, possivelmente devido à menor mobilidade das cadeias poliméricas com a presença das cargas e à estrutura química da celulose com suas fortes ligações de hidrogênio. A adição de cargas reduz a resistência ao impacto do PEAD devido às restrições impostas à mobilidade molecular do polímero. Novamente o uso do anidrido maleico proporcionou um melhor resultado em relação ao silano.

Fica evidente o efeito do pó de madeira de Pinus taeda, uma carga de fonte natural e renovável, na melhoria das propriedades mecânicas dos compósitos poliméricos em relação ao polímero puro.

\section{Referências}

1. Jawaid, M., \& Abdul Khalil, H. P. S. (2011). Cellulosic/ synthetic fibre reinforced polymer hybrid composites: A review. Carbohydrate Polymers, 86(1), 1-18. http://dx.doi. org/10.1016/j.carbpol.2011.04.043.

2. Cândido, G. M., Rezende, M. C., Donadon, M. V., \& Almeida, S. F. M. (2012). Fractografia de compósito estrutural aeronáutico submetido à caracterização de tenacidade à fratura interlaminar em modo I. Polímeros: Ciência e Tecnologia, 22(1), 41-53. http://dx.doi.org/10.1590/S0104-14282012005000019.

3. Ku, H., Wang, H., Pattarachaiyakoop, N., \& Trada, M. (2011). A review on the tensile properties of natural fiber reinforced polymer composites. Composites Part B: Engineering, 42(4), 856-873. http://dx.doi.org/10.1016/j.compositesb.2011.01.010.

4. Pöllänen, M., Suvanto, M., \& Pakkanen, T. T. (2013). Cellulose reinforced high density polyethylene composites - morphology, mechanical and thermal expansion properties. Composites Science and Technology, 76, 21-28. http://dx.doi.org/10.1016/j. compscitech.2012.12.013.

5. Zimmermann, M. V. G., Turella, T. C., Zattera, A. J., \& Santana, R. M. C. (2014). Influência do tratamento químico da fibra de bananeira em compósitos de poli(etileno-co-acetato de vinila) com e sem agente de expansão. Polímeros: Ciência e Tecnologia, 24(1), 58-64. http://dx.doi.org/10.4322/polimeros.2013.071.

6. Hablitzel, M. P., Garcia, D. E., \& Hotza, D. (2011). Interfaces fracas em compósitos de matriz cerâmica de alumina/alumina. Revista Matéria, 16(3), 788-794. http://dx.doi.org/10.1590/ S1517-70762011000300006.

7. Gregolin, E. N., Goldenstein, H., Gonçalves, M., \& Santos, R. G. (2002). Aluminium matrix composites reinforced with co-continuous interlaced phases aluminium-alumina needles. Materials Research, 5(3), 337-342. http://dx.doi.org/10.1590/ S1516-14392002000300019.

8. Vieira, L. E., Jr., Rodrigues, J. B., No., Hotza, D., Klein, A. N. (2009). Compósitos de matriz metálica reforçados pela dispersão de partículas cerâmicas produzidos por mecanossíntese: uma revisão. Exacta, 7(2), 195-204. http://dx.doi.org/10.5585/ exacta.v7i2.1626.

9. Valente, M., Sarasini, F., Marra, F., Tirillo, J., \& Pulci, G. (2011). Hybrid recycled glass fiber/wood flour thermoplastic composites: manufacturing and mechanical characterization. Composites Part A: Applied and Manufacturing, 42(6), 649657. http://dx.doi.org/10.1016/j.compositesa.2011.02.004.
10. Deka, B. K., \& Maji, T. K. (2011). Study on the properties of nanocomposite based on high density polyethylene, polypropylene, polyvinyl chloride and wood. Composites Part A: Applied and Manufacturing, 42(6), 686-693. http:// dx.doi.org/10.1016/j.compositesa.2011.02.009.

11. Mareri, P., Bastide, S., Binda, N., \& Crespy, A. (1998). Mechanical behavior of polypropylene composites containing fine mineral filler: effect of filler surface treatment. Composites Science and Technology, 58(5), 747-752. http://dx.doi.org/10.1016/ S0266-3538(97)00156-5.

12. John, M. J., \& Thomas, S. (2008). Biofibres and biocomposites. Carbohydrate Polymers, 71(3), 343-364. http://dx.doi. org/10.1016/j.carbpol.2007.05.040.

13. Xie, Y., Hill, C. A. S., Xiao, Z., Militz, H., \& Mai, C. (2010). Silane coupling agents used for natural fiber/polymer composites: a review. Composites Part A: Applied and Manufacturing, 41(7), 806-819. http://dx.doi.org/10.1016/j.compositesa.2010.03.005.

14. Silva, L. J., Panzera, T. H., Velloso, V. R., Christoforo, A. L., \& Scarpa, F. (2012). Hybrid polymeric composites reinforced with sisal fibres and silica microparticles. Composites Part B: Engineering, 43(8), 3436-3444. http://dx.doi.org/10.1016/j. compositesb.2012.01.026.

15. Faruk, O., \& Matuana, L. M. (2008). Nanoclay reinforced HDPE as a matrix for wood-plastic composites. Composites Science and Technology, 68(9), 2073-2077. http://dx.doi. org/10.1016/j.compscitech.2008.03.004.

16. Fabiyi, J. S., \& McDonald, A. G. (2010). Effect of wood species on property and weathing performance of wood plastic composites. Composites Part A: Applied and Manufacturing, 41(10), 14341440. http://dx.doi.org/10.1016/j.compositesa.2010.06.004.

17. Kabir, M. M., Wang, H., Lau, K. T., \& Cardona, F. (2012). Chemical treatments on plant-based natural fibre reinforced polymer composites: An overview. Composites Part B: Engineering, 43(7), 2883-2892. http://dx.doi.org/10.1016/j. compositesb.2012.04.053.

18. Correa, C. A., Fonseca, C. N. P., Neves, S., Razzino, C. A., \& Hage, E., Jr. (2003). Compósitos termoplásticos com madeira. Polímeros: Ciência e Tecnologia, 13(3), 154-165. http://dx.doi. org/10.1590/S0104-14282003000300005.

19. Vianna, W. L., Correa, C. A., \& Razzino, C. A. (2004). Efeitos do tipo de poliestireno de alto impacto nas propriedades de compósitos termoplásticos com farinha de resíduo de madeira. Polimeros: Ciência e Tecnologia, 14(5), 339-348. http://dx.doi. org/10.1590/S0104-14282004000500012.

20. Redighieri, K. I., \& Costa, D. A. (2008). Compósitos de polietileno reciclado e partículas de madeira de reflorestamento tratadas dom polietileno modificado. Polímeros: Ciência e Tecnologia, 18(1), 5-11. http://dx.doi.org/10.1590/S010414282008000100006 . 